\title{
Optical Emission from the Interaction of Fast Electrons with Metallic Films Containing a Circular Aperture: A Study of Radiative Decoherence of Fast Electrons
}

\author{
F. J. García de Abajo \\ Instituto de Óptica-CSIC, Serrano 121, 28006 Madrid, Spain
}

(Received 1 September 2008; published 11 June 2009)

\begin{abstract}
Light emission resulting from the interaction of swift electrons with a distant material is shown to produce an unexpectedly large fraction of decoherence in the moving charges. The decoherence probability diverges for an electron passing through a hole drilled in a perfectly conducting metal film, regardless of the size of the opening. This divergence, which is logarithmic in the ratio of film radius to aperture radius, originates in an infrared catastrophe that differs from other sources of decoherence (e.g., bremsstrahlung radiation). Our results provide new avenues for controlling and assessing the role of coherence during electron acceleration (for example, in transmission electron microscopes) and for exploiting partial quantum interference of fast electrons.
\end{abstract}

DOI: 10.1103/PhysRevLett.102.237401

Swift electrons undergo inelastic losses when interacting with absorbing materials, but they can also lose energy by emitting photons. The analysis of these processes is actually an excellent source of information on the optical response of nanostructures, based on which such techniques as electron energy-loss spectroscopy $[1,2]$ and cathodoluminescence [3] enable plasmons and other localized excitations to be sampled with impressive spatial resolution. Interestingly, the radiative loss channel can be significant even if the electron beam is not intersecting any material, a phenomenon that has been termed diffraction radiation emission [4]. In this context, it is relevant for us that energy losses, however minute, produce decoherence in the quantum state of the charged projectile because the initial state is different from the final state involving material excitations or emitted photons. This problem is framed in the active field of particle decoherence produced by coupling to quantum baths [5] and introduces a unique channel for that coupling.

In this Letter, we demonstrate that the decoherence probability diverges for an electron passing through a perfectly conducting metal-film opening of arbitrarily large diameter. For films of finite extension, the divergence grows logarithmically with the ratio of film radius to aperture radius. Similarly to the Bloch-Nordsieck infrared catastrophe [6], low frequencies $\omega$ produce a divergent $\sim 1 / \omega$ contribution to decoherence. Fully nonretarded algebraic analysis also predicts a diverging decoherence probability when the electron velocity approaches the speed of light. The representative examples presented here can contribute to understand the role of decoherence during image formation in transmission electron microscopy (for instance, in the Stobbs factor [7]) and to provide a new framework for studying electron beams with controlled degree of coherence.

Absorption and radiative decoherence probabilities.The energy loss $(\Delta E>0)$ suffered by a fast electron passing near an inhomogeneous sample and moving with con-
PACS numbers: 78.70. $-\mathrm{g}, 41.60 .-\mathrm{m}, 78.20 . \mathrm{Bh}, 79.20 . \mathrm{Uv}$

stant velocity $\mathbf{v}$ along a straight line trajectory $\mathbf{r}=\mathbf{r}_{e}(t)$ can be related to the force exerted by the induced electric field $\mathbf{E}^{\text {ind }}$ acting back on the electron as [8]

$$
\Delta E=e \int d t \mathbf{v} \cdot \mathbf{E}^{\mathrm{ind}}\left[\mathbf{r}_{e}(t), t\right]=\int_{0}^{\infty} \hbar \omega d \omega(d P / d \omega),
$$

where

$$
\frac{d P}{d \omega}=\frac{e}{\pi \hbar \omega} \int d t \operatorname{Re}\left\{e^{-i \omega t} \mathbf{v} \cdot \mathbf{E}^{\text {ind }}\left[\mathbf{r}_{e}(t), \omega\right]\right\}
$$

is the differential loss probability per unit frequency. Here, we focus on the overall decoherence probability

$$
P=\int_{0}^{\infty} d \omega(d P / d \omega)
$$

Part of the energy-loss events originate in radiative processes, the likelihood of which corresponds to the integral of the Poynting vector over a sphere of large radius centered at the interaction region. The differential probability of emitting one photon of frequency $\omega$ becomes

$$
\frac{d P^{\mathrm{rad}}}{d \omega}=\frac{c}{(2 \pi)^{2} \hbar \omega} \int d \Omega|\mathbf{f}(\Omega, \omega)|^{2},
$$

where $\mathbf{f}(\Omega, \omega)$ is the electric field amplitude along the farfield direction $\Omega$. Obviously, $d P^{\mathrm{rad}} / d \omega$ cannot exceed $d P / d \omega$, but the two probabilities are equal for nonabsorbing materials, such as the perfect-conductor structures that we discuss below, for which the equality $P=P^{\text {rad }}$ is explicitly verified. We further show how $P$ can diverge for a hole drilled in a film of infinite extension, meaning that an infinite number of photons is emitted by the electronaperture coupling, although the combined energy of all photons remains finite and the coupling itself is actually weak in large openings. The fraction of electrons undergoing inelastic scattering $f$ is thus related to $P$ through $f=$ $1-\exp (-P)$.

The probabilities of Eqs. (1) and (3) have the form [6]

$$
d P / d \omega=F / \omega
$$


where the absolute magnitude of the light frequency enters $F$ only through the spectral dependence of the permittivity. That is, all distances characterizing the system (e.g., a diameter) appear divided by the wavelength $\lambda$. This observation has two important consequences.

(1) The integrated probabilities $P$ and $P^{\text {rad }}$ are invariant under scaling of the geometry if the dielectric properties of the involved materials are not changed (for instance, for good conductors and insulators, which have spectrally flat permittivities at long wavelengths).

(2) Certain self-similar shapes must produce a divergence in $P$ when $F$ remains finite at long wavelengths (infrared catastrophe [6]). Here are two examples: (i) an electron passing through a hole drilled in a metal film, in which the long-wavelength behavior is like that of an electron passing through a homogeneous film (i.e., aperture radius $\ll \lambda$ ), and (ii) an electron passing near a wedge or any variant of a wedge (e.g., a semi-infinite screen). We examine case (i) in detail further below.

In the planar, perfectly conducting screen, the electron decoherence probability under normal incidence, corresponding to the spectral yield of transition radiation [9], satisfies Eq. (4) with

$$
F=F_{\text {film }}=\frac{1}{\pi \beta} \frac{e^{2}}{\hbar c}\left[\left(1+\beta^{2}\right) \log \left(\frac{1+\beta}{1-\beta}\right)-2 \beta\right],
$$

where $\beta=v / c$. Incidentally, $F_{\text {film }}$ is independent of photon frequency, and therefore $P$ diverges due to both long and short wavelength contributions.

Decoherence by a sphere.-We first study the decoherence produced by a spherical particle. At large separations from the electron beam, only dipolar polarization contributes efficiently, driven by the external field

$$
\mathbf{E}^{\mathrm{ext}}(\mathbf{r}, \omega)=\frac{2 e \omega}{v^{2} \gamma} e^{i \omega z / v}\left[\frac{i}{\gamma} K_{0}\left(\frac{\omega R}{v \gamma}\right) \hat{\mathbf{z}}-K_{1}\left(\frac{\omega R}{v \gamma}\right) \hat{\mathbf{R}}\right],
$$

where $\gamma=1 / \sqrt{1-\beta^{2}}, \mathbf{r}=(\mathbf{R}, z)$, with $\mathbf{R}=(x, y)$, is given relative to the position of the electron at time $t=$ 0 , and the velocity is assumed to be along the positive $z$ axis. This leads to the following closedform expression for the loss probability [10]:

$$
\begin{aligned}
\frac{d P}{d \omega}= & \frac{4 e^{2} \omega^{2}}{\pi \hbar v^{4} \gamma^{2}}\left[\left(K_{1}^{2}+\frac{1}{\gamma^{2}} K_{0}^{2}\right) \operatorname{Im}\left\{\alpha^{E}\right\}\right. \\
& \left.+\frac{v^{2}}{c^{2}} K_{1}^{2} \operatorname{Im}\left\{\alpha^{M}\right\}\right],
\end{aligned}
$$

where $\alpha^{E}$ and $\alpha^{M}$ are the electric and magnetic polarizabilities of the sphere. The modified Bessel functions $K_{0}$ and $K_{1}$ decay exponentially with their argument $\omega b / v \gamma$ in Eq. (7), where $b$ is the distance between the particle center and the electron beam [see inset of Fig. 1(a)]. Thus, only low frequencies $\omega \lesssim \gamma v / b$ are relevant, involving photons of wavelength $\lambda$ comparable to $b$ for relativistic velocities. Consequently, metal spheres behave as perfect conductors if $b$ is above several micrometers [11], leading to $\operatorname{Im}\left\{\alpha^{E}\right\}=2 \omega^{3} a^{6} / 3 c^{3}$ and $\operatorname{Im}\left\{\alpha^{M}\right\}=\omega^{3} a^{6} / 6 c^{3} \quad$ [13], where $a$ is the sphere radius. The integral of Eq. (2) then reduces to

$$
P=\frac{16}{15 \pi} \frac{e^{2}}{\hbar c}\left(\frac{a}{b}\right)^{6} \beta^{2} \gamma^{2}\left[\frac{8}{3}+4 \gamma^{2}+\beta^{2} \gamma^{2}\right]
$$

The last term inside the square brackets is due to magnetic polarization and contributes $<20 \%$.

A first remark to be made in view of the results of Fig. 1 is that the decoherence produced by a distant sphere can be observable even for macroscopic radius $a$ and impact parameter $b$. For instance, a $200-\mathrm{keV}$ electron $(v \approx 0.7 c)$ passing $1 \mathrm{~mm}$ away from the surface of a particle with $a=$ $10 \mathrm{~mm}$ experiences a $0.4 \%$ decoherence probability.

Interestingly, the long-distance asymptotic formula (8) works extremely well at low velocities compared to the full solution of Maxwell's equations including all multipoles [10], down to $b \approx a$ [cf. dashed curves and solid curves in Fig. 1(b)]. Furthermore, the external electric field ap-
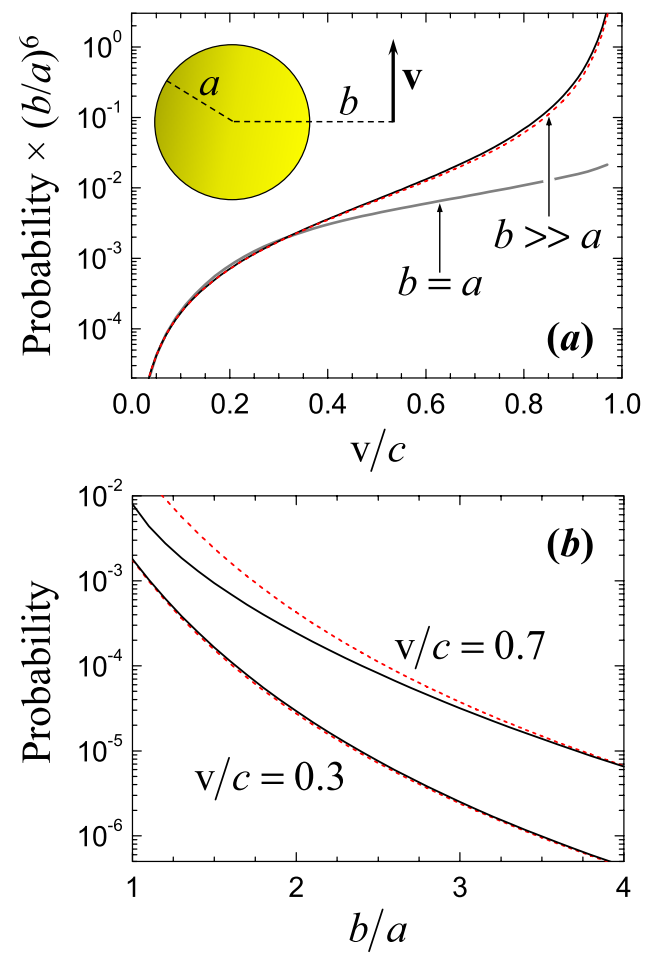

FIG. 1 (color online). Decoherence by a metallic sphere. (a) Probability of photon emission by interaction of an electron with a distant perfect-conductor sphere $(b \gg a)$, as obtained from Eq. (8). The probability contribution arising from electric polarization (dashed curve) is compared to the full probability (electric and magnetic polarization, solid curve). The probability for a grazing trajectory $(b=a)$ is also plotted for comparison (gray curve), calculated with full inclusion of all multipoles [10]. (b) Dependence of the emission probability on impact parameter for two different electron velocities. The rigorous solution of Maxwell's equations (solid curves) is compared to the large $b / a$ approximation of Eq. (8) (dashed curves). 
proaches $-(2 e / v b) \hat{\mathbf{R}}$ in the $v \rightarrow c$ limit [see Eq. (6)], so that a frequency-independent dipole is induced in the particle, giving rise to a remarkable increase in decoherence probability [see $b \gg a$ curves in Fig. 1(a)]. However, the value of $b / a$ above which Eq. (8) is valid increases as $v$ approaches $c$. The sharp divergence that it predicts in the $v \rightarrow c$ limit is really an artifact of the long-wavelength approximation employed for the particle polarizability $\left(\operatorname{Im}\{\alpha\} \propto \omega^{3}\right)$, which is corrected by rigorously solving Maxwell's equations for $P$ [see $b=a$ curve in Fig. 1(a)].

Decoherence by a hole.-A situation of more physical significance corresponds to an electron passing through an opening, as depicted in Fig. 2(a). We assume perfectconductor boundary materials, and we emphasize again that this approximation is very accurate for good metals and values of the hole radius above a few microns [11]. We just address the contribution to decoherence originating in the aperture and consider the waveguide to be long (so that there are not cavity-end effects), but not so long as to contribute with absorption losses in the straight sections of the guide. Obviously, the waveguide ends produce further decoherence that would require a similar analysis to the one presented next. We study this system by solving Maxwell's equations using a modal expansion of the electric field in the cylindrical sections above $(n=1)$, inside $(n=2)$, and below $(n=3)$ the hole. The electric field is obtained by finding the coefficients $\beta_{n j}$ of the modal expansions $\mathbf{E}=\mathbf{E}_{n}^{\text {inc }}+\sum_{j} \beta_{n j} \mathbf{E}_{n j}$ inside the three noted cylindrical sections, described by the complete basis sets of modes $\mathbf{E}_{n j}$. The radii of these sections $\left(R_{n}\right)$ are taken as $R_{1}=R_{3}=R_{o}$ outside the aperture and $R_{2}=R_{i}$ inside it [see Fig. 2(a)]. The electric field of the incident electron $\mathbf{E}_{n}^{\text {inc }}$ is given by Eq. (6) after replacing $K_{\nu}$ by $K_{\nu}+D_{n} J_{\nu}$, where $D_{n}=-K_{\nu}\left(\omega R_{n} / v \gamma\right) / J_{\nu}\left(\omega R_{n} / v \gamma\right)$. The $D_{n} J_{\nu}$ term, originating in the reflection of the external field at the wall of an infinite cylindrical cavity of radius $R_{n}$, guarantees that the azimuthal and $z$ components of $\mathbf{E}_{n}^{\text {inc }}$ vanish at the metal boundary. For simplicity, we consider axial electron trajectories, which only couple to modes of $\nu=0$ azimuthal symmetry and $p$ polarization [14]:

$$
\mathbf{E}_{n j}(\mathbf{r})=\frac{c}{\omega}\left[-i q_{n j} J_{1}\left(Q_{n j} R\right) \hat{\mathbf{R}}+Q_{n j} J_{0}\left(Q_{n j} R\right) \hat{\mathbf{z}}\right] e^{i q_{n j} z},
$$

where $j$ labels modes determined by the condition $J_{0}\left(Q_{n j} R_{n}\right)=0$, and the $z$ component of the wave vector is given by $q_{n j}= \pm \sqrt{\omega^{2} / c^{2}-Q_{n j}^{2}}$ (the sign is + above the film, - below the film, and \pm inside the hole). Following a similar method of solution as for light transmission through individual holes [15], we use the continuity of the $x$ and $y$ components of the electric field to express the coefficients $\beta_{1 j}$ and $\beta_{3 j}$ in terms of coefficients $\beta_{2 j}$ (inside the hole). Finally, the continuity of the magnetic field leads to a set of linear equations involving $\beta_{2 j}$. We achieve convergence in the results shown in Fig. 2 by using $\sim 20$ modes inside the hole and $\sim 200$ modes outside it for the finite values of $R_{o} / R_{i}$ under consideration. Infinite

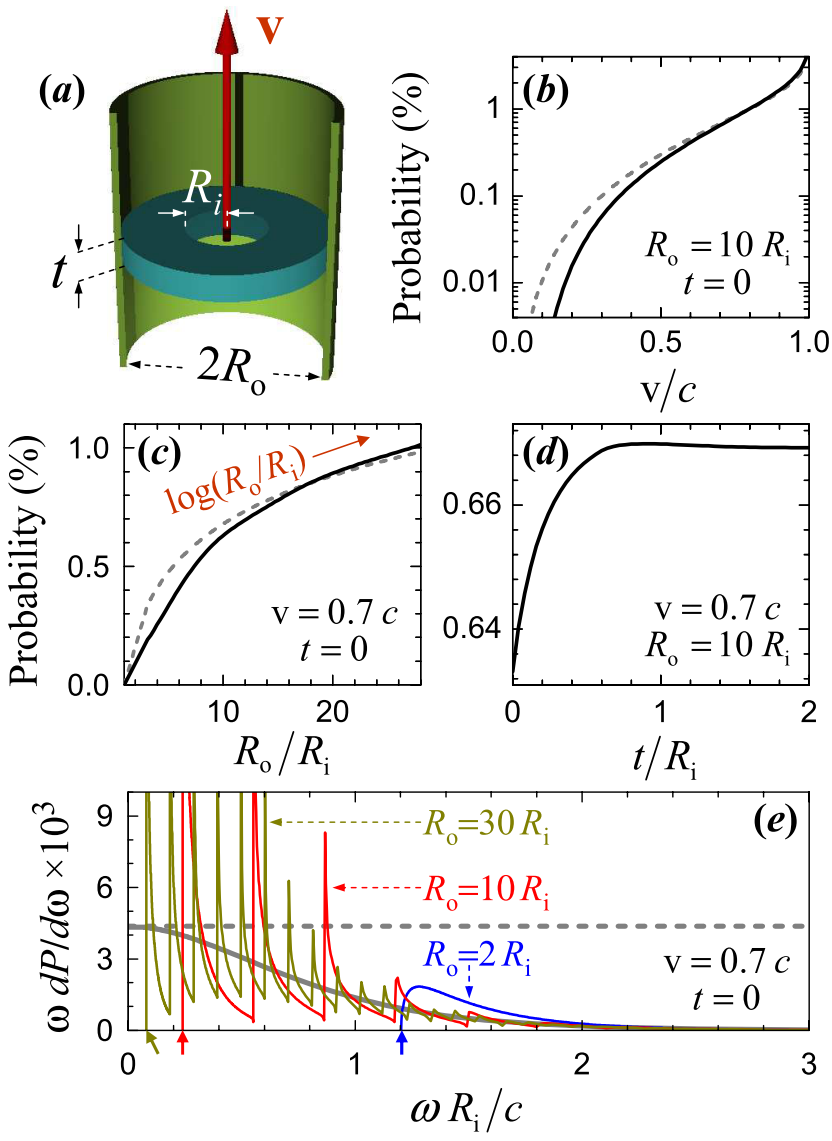

FIG. 2 (color online). Decoherence by a circular aperture. (a) Schematic representation of an electron moving with velocity $v$ along the axis of a cylindrical waveguide of radius $R_{o}$ and crossing a circular hole in an internal film. The waveguide is infinite in length, but only a finite piece of it is shown, with the side towards the viewer cut open, so that the interior film of thickness $t$ with the opening of radius $R_{i}$ is visible. (b)(d) Dependence of the decoherence probability on electron velocity (b), the ratio of waveguide radius to hole radius (c), and hole thickness (d) for choices of geometrical parameters as shown by text insets. Solid curves are rigorous solutions of Maxwell's equations. Dashed curves in (b) and (c) correspond to the analytical approximation of Eq. (9). (e) Frequencyresolved photon emission probability for various values of the $R_{o} / R_{i}$ ratio. The $R_{o} \rightarrow \infty$ limit is shown for comparison as a solid gray curve. The dashed line represents the emission for an unperforated film [Eqs. (4) and (5)].

films $\left(R_{o} / R_{i} \rightarrow \infty\right)$ require $>1000$ modes outside the film. In both cases, the numerical values of the loss probability calculated from Eq. (1) agree within $<0.1 \%$ with the photon emission flux, obtained from Eq. (3) for infinite films and from $d P^{\mathrm{rad}} / d \omega=\sum_{j} P_{j}\left(\left|\beta_{1 j}\right|^{2}+\left|\beta_{3 j}\right|^{2}\right)$, for finite $R_{o}$, where $P_{j}=\left[\left(R_{o} c / \omega\right)^{2} / 4 \pi \hbar\right] \operatorname{Re}\left\{q_{1 j}\right\} J_{1}^{2}\left(Q_{1 j} R_{o}\right)$ is the photon flux of mode $j$.

The spectral dependence of the photon emission exhibits pronounced maxima at the onset of guided modes [see Fig. 2(e)], while no losses are permitted below the threshold for the first $p$ guided mode (see arrows in the abscissas, 
corresponding to $\omega / c \approx 2.405 / R_{o}$ ), thus averting the infrared catastrophe, which is a common characteristic of finite systems ( $\lambda \gg$ size). The spectrum slowly evolves towards that of an infinite film (gray curve) for increasing cylindrical-cavity radius $R_{o}$. As noted above, the loss probability in an infinite film with and without a hole approaches the same diverging limiting value at small $\omega$ 's, giving rise to an infinite decoherence probability (but also to a finite average energy loss in the perforated film). This is in contrast to the sphere considered above, in which $d P / d \omega=0$ at $\omega=0$. The drilled film modifies the radiation field producing effective electron-photon coupling with finite $F$ at $\omega=0$. This is similar to bremsstrahlung emission [16], but with a completely different mechanism that does not require strong Coulomb interaction to deflect the electron.

As a rule of thumb, we expect light components of wavelength in the $R_{i}<\lambda<R_{o}$ range to effectively see a film without a hole [15]. This intuitive picture is consistent with the $R$ dependence of the electron field in Eq. (6). Integrating Eq. (4) over the corresponding range of frequencies, with $F_{\text {film }}$ [Eq. (5)] substituted for $F$, we find

$$
P=C F_{\text {film }} \log \left(R_{o} / R_{i}\right),
$$

where one would expect a correction factor $C \sim 1$. We show in Figs. 2(b) and 2(c) rigorous modal-expansion calculations of $P$ (solid curves), in remarkable agreement with Eq. (9) for $C=0.75$ (dashed curves). As anticipated, the probability displays a logarithmic divergence $\left[\sim \log \left(R_{o} / R_{i}\right)\right]$ for large film radius [Fig. 2(c)]. This is consistent with the observed weak dependence on film thickness [see Fig. 2(d); also notice that Eq. (5) is independent of that parameter].

The divergence of $F_{\text {film }} \approx-\left(2 e^{2} / \pi \hbar c\right) \log (1-v / c)$ in the $v \rightarrow c$ limit is inherited by the decoherence due to the hole, as shown in Fig. 2(b). This is a consequence of the fact that $\mathbf{E}^{\text {ext }}$ does not depend on $\omega$ in that limit.

In a thin film, photons are preferentially emitted along directions relatively grazing with respect to the filmsurface plane [12]. Light generation encompasses components of the electron-radiation system in which mutual coherence is lost for different values of the momentum transfer, because they involve orthogonal photon quantum states. This can be experimentally corroborated by recording the interference pattern of electrons transmitted through a hole. For instance, different fringe-pattern contrast is expected in Young's double slit experiment after transmitting the beam through apertures of different dimensions, subject to the condition that the momentum transferred by the hole $\sim \hbar / R_{i}$ be a sizable fraction of the momentum transferred by the slits $\sim \hbar / d$, where $d$ is the slit separation.

In conclusion, we have demonstrated that swift electrons can couple with high efficiency to distant objects (for example, when passing through a wide opening in a film), thus producing significant decoherence in its quantum state, and particularly if the beam has to cross various diaphragms along its path, as is usually the case in electron microscopes. The above theory applies even to macroscopic distances. The decoherence probability is actually divergent for a hole of arbitrarily large, finite radius drilled in a metal film of infinite extension, but it also diverges near finite objects when the electron velocity approaches the speed of light. These results should be useful both in the design of probing beams with any desired degree of coherence for studies of electron interference phenomena and in the assessment of the role played by radiative decoherence during image formation in transmission electron microscopy. In particular, this study could shed some light into the origin of the Stobbs factor [7]. A conclusive explanation for this effect is still missing, although recent progress in the role of inelastic collisions has been recently reported [17]. We hope that the theoretical predictions elaborated here can stimulate further experimental efforts in this direction.

I thank Archie Howie and Florian Marquardt for valuable discussions. Work supported by the Spanish MICINN (MAT2007-66050 and Consolider NanoLight.es) and by the EU (NMP4-2006-016881-SPANS and NMP4-SL2008-213669-ENSEMBLE).

[1] J. Nelayah et al., Nature Phys. 3, 348 (2007).

[2] M. Bosman et al., Nanotechnology 18, 165505 (2007).

[3] N. Yamamoto et al., Phys. Rev. B 64, 205419 (2001).

[4] P. Karataev et al., Phys. Rev. Lett. 93, 244802 (2004).

[5] F. Marquardt, arXiv:cond-mat/0604626v1.

[6] F. Bloch and A. Nordsieck, Phys. Rev. 52, 54 (1937).

[7] M. J. Hÿtch and W. M. Stobbs, Ultramicroscopy 53, 191 (1994); A. Howie, Ultramicroscopy 98, 73 (2004).

[8] R. H. Ritchie, Phys. Rev. 106, 874 (1957).

[9] V. L. Ginzburg and I. M. Frank, Zh. Eksp. Teor. Fiz. 16, 15 (1946).

[10] F. J. García de Abajo, Phys. Rev. B 59, 3095 (1999).

[11] We have contrasted some of the results shown here with generalized Mie calculations using the actual dielectric function of gold. Perfect-conductor behavior is achieved for electron-metal distances above $2 \mu \mathrm{m}$ [12].

[12] See EPAPS Document No. E-PRLTAO-103-053952 for auxiliary material. For more information on EPAPS, see http://www.aip.org/pubservs/epaps.html.

[13] The optical theorem leads to $\operatorname{Im}\{\alpha\}=\left(2 \omega^{3} / 3 c^{3}\right)|\alpha|^{2}$ for both electric and magnetic polarizabilities in nonlossy particles. Additionally, perfectly conducting spheres satisfy $\alpha^{E}=-2 \alpha^{M}=a^{3}$ in the long-wavelength limit.

[14] F. J. García de Abajo et al., Phys. Rev. B 68, 205105 (2003).

[15] A. Roberts, J. Opt. Soc. Am. A 4, 1970 (1987).

[16] J.D. Jackson, Classical Electrodynamics (Wiley, New York, 1999).

[17] K. A. Mkhoyan et al., Phys. Rev. Lett. 100, 025503 (2008). 